\title{
ABORDAGEM TERAPÊUTICA NA EXACERBAÇÃO DA DOENÇA PULMONAR OBSTRUTIVA CRÔNICA (DPOC)
}

\author{
MANAGEMENT IN EXACERBATIONS OF CHRONIC OBSTRUCTIVE PULMONARY DISEASE (COPD)
}

Marcos C. Borges ${ }^{1}$; Elcio S. O. Vianna² \& João Terra Filho ${ }^{3}$

${ }^{1}$ Médico Colaborador e Pós Graduando. ${ }^{2}$ Docente. Divisão de Pneumologia. Departamento de Clinica Médica. Faculdade de Medicina de Ribeirão Preto - USP.

Correspondencia: Prof. Dr. João Terra Filho. Divisão de Pneumologia. Departamento de Clincia Médica. Faculdade de Medicina de Ribeirão Preto - USP. Campus Universitário - CEP 14.048-900 - Ribeirão Preto - SP. e-mail: jtfilho@fmrp.usp.br

BORGES MC; VIANNA ESO \& TERRA FILHO J. Abordagem terapêutica na exacerbação da doença pulmonar obstrutiva crônica (DPOC). Medicina, Ribeirão Preto, 36: 241-247, abr./dez. 2003.

RESUMO - Exacerbações de DPOC causam aumento da morbimortalidade, internação, piora da qualidade de vida e custos para o paciente e para o sistema de saúde. O paciente com DPOC apresenta, aproximadamente,duas a três exacerbações ao ano, principalmente no inverno, causando progressiva deterioração fisiológica e aumento da inflamação das vias aéreas. O tratamento se faz com broncodilatadores, corticosteróides, oxigenoterapia, ventilação mecânica e antibióticos, em alguns casos.

UNITERMOS - Pneumopatias Obstrutivas.

\section{1- INTRODUÇÃO}

A doença pulmonar obstrutiva crônica (DPOC) é importante causa de morbimortalidade por doenças crônicas, em todo mundo, sendo, segundo a OMS, a quarta principal causa de morte. Além disso, aumentos na prevalência e na mortalidade podem ser previstos para as próximas décadas.

A DPOC é definida pela presença de obstrução ao fluxo aéreo, progressiva, não totalmente reversível, secundária à bronquite crônica, ao enfisema pulmonar ou a ambos. Em aproximadamente 60 a $80 \%$ dos pacientes, a hiper-responsividade brônquica está presente. A limitação ao fluxo aéreo é causada tanto pela inflamação das pequenas vias aéreas, causando remodelamento e estreitamento delas, quanto pela perda das conexões alveolares e redução do recolhimento elástico, secundário à destruição do parênquima pulmonar.

O enfisema é definido, anatomicamente, como um alargamento anormal, permanente, dos espaços aéreos distais aos bronquíolos terminais, acompanhado de destruição de suas paredes, sem fibrose óbvia. A bronquite crônica é definida, clinicamente, pela presença de tosse produtiva, pelo menos, três meses por ano, em dois anos consecutivos, afastando outras causas de expectoração crônica.

A história clínica de tosse, geralmente produtiva, dispnéia e sibilos, progressiva, associada à presença de fatores de risco, como tabagismo (80\%), gases tóxicos, poluição, infecção, exposição a fogão de lenha ou deficiência de alfa-1 antitripsina, na exclusão de outras doenças pulmonares, é suficiente para o diagnóstico. A avaliação da função pulmonar confirma o diagnóstico e auxilia na classificação da gravidade. A presença de uma relação VEF1/CVF menor que 0,75 ou menor que $90 \%$ do previsto, associada ou não a uma redução do VEF1, confirma a obstrução de vias aéreas.

Com prevalência de $5 \%$ na população adulta e estimativa de 7,5 milhões de doentes no Brasil, a DPOC tem significativa morbimortalidade, comprometimento 
da qualidade de vida, da produtividade e alto custo social. O tratamento tem como objetivos reduzir a mortalidade, obter melhora funcional e da qualidade de vida, redução das exacerbações e das internações.

\section{2- EXACERBAÇÕES DA DPOC}

\section{1- Características clínicas}

As exacerbações dos sintomas respiratórios, que evoluem com a necessidade de intervenção médica são eventos clínicos importantes na história dos pacientes com DPOC, apresentando relevante impacto social e econômico.

O paciente com DPOC apresenta aproximadamente duas a três exacerbações ao ano, dependendo da gravidade da doença, sendo mais freqüentes no inverno. A exacerbação aguda da DPOC manifestase com diferentes formas clínicas e gravidades. O quadro clínico mais freqüente é o aumento da dispnéia. Pode ocorrer, também, aumento da tosse, aumento e/ ou alteração do aspecto da expectoração, sibilos, opressão torácica, indisposição, fadiga e diminuição na tolerância aos exercícios. Irritabilidade, tremores, confusão mental, sonolência, coma e convulsão podem estar presentes nos casos mais graves. A febre nem sempre é presente.

A infecção da árvore traqueobrônquica é a causa mais freqüente das exacerbações, podendo chegar até a $50 \%$ dos casos. Dos quadros infecciosos, 2/3 podem ser bacterianos e 1/3 viral, sendo o Rinovírus, Haemophilus influenzae, Streptococcus pneumoniae e Moraxella catarrhalis os agentes mais encontrados. Outras causas que devem ser investigadas são: poluição do ar, pneumonia, tromboembolismo pulmonar, pneumotórax, uso de sedativos ou outras drogas (ex.: betabloqueadores), arritmias cardíacas, insuficiência cardíaca direita ou esquerda, trauma torácico, aspiração e/ou refluxo gastroesofágico e deterioração da doença de base. (Tabela I) Cerca de $1 / 3$ das causas das exacerbações graves não são identificadas.

\section{2- Avaliação da gravidade}

A avaliação da gravidade se faz através da história clínica (atual e prévia), exame físico e exames laboratoriais. Na história clínica, deve-se avaliar a freqüência e a gravidade das exacerbações atuais e prévias; presença de dispnéia, tosse e característica da expectoração; duração e progressão dos sintomas; repercussão da exacerbação nas atividades diárias, como tolerância ao exercício; alteração do sono e do comportamento; presença de co-morbidades e condição socioeconômica.

No exame físico, são sinais de gravidade o uso de musculatura acessória, respiração paradoxal, sinais clínicos de cor pulmonale, instabilidade hemodinâmica, estado mental alterado, cianose e presença de outras co-morbidades. Na gasometria, avalia-se a presença de hipoxemia, hipercapnia e acidose, sendo muito importante a comparação com um exame prévio, pois uma alteração aguda é mais importante que o valor absoluto. Entretanto, se a $\mathrm{PaO} 2$ for menor que $50 \mathrm{mmHg}$, a $\mathrm{PaCO} 2$ maior que $70 \mathrm{mmHg}$ e o $\mathrm{pH}$ menor que 7,30 , a monitorização do paciente deve ser cuidadosa.

O estudo radiológico do tórax pode contribuir para a identificação da causa do episódio de exacerbação ou, alternativamente, para outros diagnósticos, que podem simular os mesmos sintomas. O eletrocardiograma auxilia no diagnóstico de sobrecarga ventricular direita, episódios isquêmicos do miocárdio e arritmias, que podem estar presentes nos pacientes, em consequiência da hipoxemia grave e da acidose associadas ao aumento da pós-carga do ventrículo direito e ao uso de drogas arritmogênicas, como digitais e broncodilatadores. Em algumas circunstâncias, o ecocardiograma pode fornecer importantes informações, como, por exemplo, estimar a pressão na artéria pulmonar.

Tabela I - Princip ais causas de exacerb ação na DPOC Causas pulmonares

- Infecção da árvore traque obrônquica

- Pneumoria

Tromboembolismo pulmonar

Pneumotórax

Poluição do ar

Trauma torácico

Deterioração da doença de base

A spiração ou reflux o gastroesofágico

Causas extrapulmonares

- Arritmias cardíacas

- Insuficiência cardiaca direita ou esquerda

- U so de sedativos ou outras drogas

(ex.: betabloque adores) 
O paciente portador de DPOC, em especial o com quadro clínico grave, apresenta vários fatores de risco para desenvolvimento de embolia pulmonar (TEP), podendo ser difícil distingui-la de uma exacerbação infecciosa. As alterações estruturais dos ventrículos e dos grandes vasos pulmonares, comuns na DPOC, complicam a interpretação dos exames habituais. Assim sendo, na suspeita de embolia pulmonar, outros métodos diagnósticos devem ser utilizados, como: dosagens de D-dímeros, angio-tomografia computadorizada e angiografia pulmonar. Em tal condição clínica, pode ser duvidosa a colaboração diagnóstica da cintilografia de ventilação/perfusão pulmonar, isto porque, na presença de insuficiência cardíaca ou de DPOC, a possibilidade de que a cintilografia pulmonar traga resultados definitivos é pequena (resultados inconclusivos); pela presença da doença de base, raramente, a cintilografia de Va/Q é normal; quando isto ocorre,o diagnóstico de TEP é muito improvável, sendo a probabilidade de apenas 2 a $4 \%$. Cabe ressaltar que a cintilografia pulmonar tem maior valor diagnóstico, quando realizada em pacientes sem alterações cardiopulmonares prévias. A presença de taquicardia inexplicada, resistente às mediadas terapêuticas, ou de significativo efeito shunt sugerem a existência de embolia pulmonar. $\mathrm{Na}$ existência de fortes indicativos de TEP, é recomendável tratar o paciente juntamente com as medidas terapêuticas, habituais para a exacerbação.

\section{3- TRATAMENTO DA EXACERBAÇÃO DA DPOC}

\section{1- Critérios de internação}

O risco de morte pela exacerbação de DPOC está relacionado à gravidade da doença, ao desenvolvimento de acidose respiratória, à presença de comorbidades graves e à necessidade de suporte ventilatório. Pacientes com essas características, freqüentemente, necessitam de supervisão médica cuidadosa, geralmente, internados.

Não existem critérios bem definidos quanto à necessidade de internação, sendo importante uma avaliação individualizada, considerando a condição basal do paciente, progressão dos sintomas, resposta ao tratamento ambulatorial, grau de insuficiência respiratória, presença de co-morbidades e condição socioeconômica.

A admissão/avaliação hospitalar deve ser levada em consideração, para pacientes que se enquadrarem nos critérios apresentados na Tabela II.
Tabeh II - Indicações para intemação de pacientes com DPOC exacerbada

- Resposta inadequada ao tratamento ambulatoriald

- Incapacidade para alimentar-se, dormir ou deambuarTI - Alteração do nível de consciênciagr

- Presença de co-morbidades (ex emplo: insuficiência cardiaca) 1

- Sintomas prolongados e progressivos $]$

- Hipoxemia refratária, hipercapria com acidose (comparare com gasometrias prévias) $I$ I

- Presença de complicações (ex.: pneumotórax, arritmias)g

- Presença de cor pulmonale sem resposta ao tratamerto ambulatorialdI

Baxo nível socioeconômico, ausência de suporte domiciliar'TI

Necessidade de realização de procedimentos imasivos, como broncoscopia.

\section{2- Tratamento farmacológico}

A terapêutica da exacerbação da DPOC se faz com a utilização de broncodilatadores, corticosteróides, oxigênio e medidas específicas para o tratamento da causa da exacerbação, como antibioticoterapia, anticoagulação, drenagem pleural e outras. É muito importante a história prévia das medicações utilizadas, para que se evitem superdosagens.

A oxigenoterapia é importante no tratamento hospitalar das exacerbações da DPOC. O objetivo é manter níveis adequados de oxigenação, ou seja, uma pressão parcial de oxigênio $(\mathrm{PaO} 2)$ maior que $60 \mathrm{mmHg}$ ou uma saturação de O2 (SatO2) maior que 90\%, o que, geralmente, é alcançado através de uma cânula nasal, que a cada litro/minuto de fluxo, fornece um aumento de aproximadamente $4 \%$ na fração inspirada de $\mathrm{O} 2$ (FiO2), sendo possível a obtenção de uma FiO2 de até $40 \%$, com um fluxo de $5 \mathrm{l} / \mathrm{min}$. Caso o paciente permaneça com hipoxemia, deve-se utilizar máscara facial, máscara de Venturi ou máscara com reservatório, com as quais é possível a obtenção de $\mathrm{FiO} 2$ de até $90 \%$. A retenção de $\mathrm{CO} 2$ pode ocorrer insidiosamente, sendo importante uma avaliação, através de gasometria, $30 \mathrm{~min}$ após o início da oxigenoterapia. Concentrações altas de $\mathrm{O} 2$ inspirado pode iniciar ou agravar a retenção de $\mathrm{CO} 2$, provavelmente, pela combinação da redução do estímulo ventilatório e pela deterioração da relação ventilação/perfusão. A nebulização de drogas com altos fluxos de $\mathrm{O} 2$ tam- 
bém pode precipitar o problema. Na presença de retenção de $\mathrm{CO} 2$ sem acidose, pode-se manter as medidas já iniciadas, porém, na vigência de acidose respiratória moderada a grave, deve-se discutir a indicação de ventilação mecânica. (Tabela III)

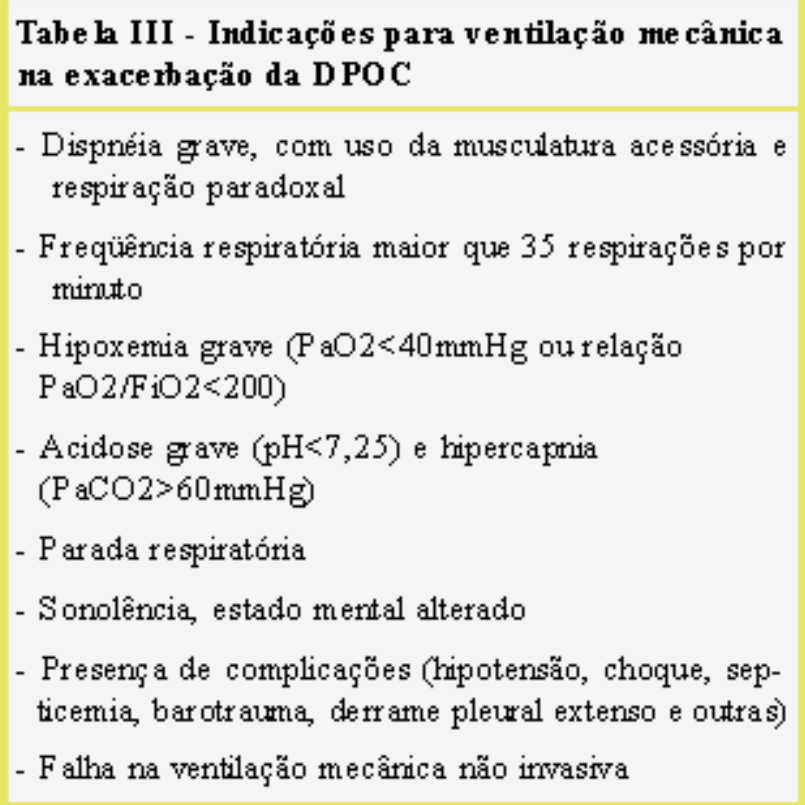

Os broncodilatadores são a base do tratamento da DPOC e sempre utilizados nas exacerbações. Betaagonistas de curta duração são as drogas broncodilatadores de escolha, devido ao rápido início de ação e efeitos colaterais bem tolerados. A via inalatória deve ser priorizada devido à ação mais rápida e presença de menos efeitos colaterais; tanto o aerosol, quanto o spray com espaçador, tem boa eficácia. Outras vias,como endovenosa e subcutânea, só devem ser utilizadas, quando a via inalatória não for efetiva, como em pacientes com alteração do estado mental, tosse excessiva ou broncoespasmo muito grave, com falência respiratória. Os betaagonistas podem ser repetidos em curtos intervalos, sendo a dose máxima determinada pela presença de efeitos colaterais, como arritmias, tremores excessivos e frequiência cardíaca maior que 140-160 bpm.

Os anticolinérgicos têm uma ação broncodilatadora inferior aos betaagonistas, porém, quando associados a eles podem ter ação sinérgica, com efeitos colaterais toleráveis. Portanto, o uso da associação entre anticolinérgicos e betaagonistas, na reversão do broncoespasmo, especialmente nos casos graves, é recomendável.
A teofilina, devido ao menor efeito broncodilatador e presença de diversos efeitos colaterais, deve ser reservada somente para casos em que não se obtém uma resposta satisfatória com a associação entre betaagonistas e anticolinérgicos ou quando não se pode utilizar a via inalatória. Pode-se utilizá-la por via oral ou endovenosa e seu nível sérico deve ser sempre monitorizado, para mantê-lo entre $10-20 \mu \mathrm{g} / \mathrm{ml}$, como forma de evitar os efeitos colaterais.

Os corticosteróides sistêmicos são benéficos no tratamento das exacerbações da DPOC, e devem se utilizados, principalmente, naqueles pacientes que necessitarem de hospitalização. Eles reduzem o período de recuperação e colaboram no restabelecimento do quadro funcional. A dose exata não está bem estabelecida, entretanto, uma dose inicial em torno de $0,5-1 \mathrm{mg} / \mathrm{kg} / \mathrm{d}$ de prednisolona ou equivalente parece ser suficiente, podendo ser mantida em torno de $72 \mathrm{~h}$, devendo, então, ser reduzida. Geralmente, não é necessário utilizá-los por um período superior a duas semanas. Tanto a via oral, quanto a via endovenosa, tem início de ação e eficácia semelhantes. O tratamento prolongado pode não resultar em maior efetividade e, por outro lado, aumenta o risco de aparecimento dos efeitos colaterais. Os principais efeitos colaterais, em uso por curto período, são hiperglicemia, hemorragia digestiva, alterações psíquicas, miopatia e complicações infecciosas.

Antibioticoterapia deve ser reservada para os pacientes com pneumonia e para aqueles que apresentarem, pelos menos, dois desses sintomas: aumento da dispnéia, alteração da cor ou aumento do volume da expectoração. Quando possível, deve-se utilizar antibióticos, de acordo com a sensibilidade do agente patogênico identificado; em caso contrário, considerar os padrões locais de sensibilidade, visando à cobertura de Haemophilus influenzae, Streptococcus pneumoniae e Moraxella catarrhalis. No tratamento, são freqüentemente utilizados: amoxicilina, amoxicilina-clavulanato, quinolonas respiratórias e macrolídeos.

\section{3- Ventilação mecânica invasiva e não invasiva}

O suporte ventilatório mecânico, não invasivo e invasivo, de modo geral, é necessário nas exacerbações agudas de DPOC com hipoxemia grave e/ou hipercapnia com acidemia, que não respondem às medidas habituais. A assistência ventilatória não invasiva é considerada um recurso terapêutico bas- 
tante eficiente no tratamento da insuficiência ventilatória crônica, decorrente de doenças que envolvem a caixa torácica, mas, quanto ao portador de DPOC, tem se mostrado útil apenas para um seleto grupo de pacientes para os quais pode ser recomendada após o esgotamento das medidas habituais. Contrastando, na presença de insuficiência ventilatória aguda, secundária às exacerbações leves (pH:7,30-7,35) e moderadas $(\mathrm{pH}<7,30)$ da DPOC, existem consideráveis evidências de seu benefício. São circunstâncias em que a prevenção da intubação, a redução da mortalidade e da falha terapêutica são as principais vantagens de seu uso precoce. A utilização da assistência ventilatória não invasiva abriu novas perspectivas para o tratamento de pacientes com insuficiência respiratória, principalmente, com relação aomomento da intervenção e às condições da área física para sua prática. A dispensa da intubação, sedação e paralisia muscular permite que seja desenvolvida fora da UTI. Por outro lado, deve ser instituída mais precocemente, no curso da doença, antes mesmo do desenvolvimento de acidose respiratória grave e da necessidade imperiosa de assistência ventilatória invasiva. Habitualmente, utilizase a pressão de suporte ou o suporte ventilatório com dois níveis de pressão (conhecido como BiPAP), através de máscara facial ou nasal, regulando-se os níveis pressóricos, para que se obtenha volume corrente satisfatório, melhora gasométrica e conforto ao paciente. No presente, algumas contra-indicações para a assistência ventilatória não invasiva são apresentadas na Tabela IV.

Para pacientes em estados mais graves, que necessitam de intubação precoce ou não responderam à assistência ventilatória não invasiva, é manda-

Tabela IV - Contra-indicações para ve utihção mecânica não invasiva, na exacerbação da D POC
Alter ação do rível de consciência
Parada respiratória
P aciente não colaborativo
- Instabilidade hemodinâtmica
Presença de secreção abundante, alto risco de aşiração
- Cirug ga facial ou gastroesofágica recente
Trauma crariofacial
O besidade extrema
Ausência de profissionais com experiência

tória a instituição da assistência ventilatória invasiva. A indicação do suporte ventilatório invasivo é, primariamente, clínica, e o maior determinante é o nível de consciência do paciente, não existindo qualquer valor absoluto de $\mathrm{PaCO} 2$ ou $\mathrm{pH}$ que o indique. Com a ventilação mecânica invasiva visa-se: fornecer repouso muscular; manter uma $\mathrm{PaCO} 2$ inicial entre 45 e $65 \mathrm{mmHg}$, sem acidose respiratória; devem ser utilizadas baixas frequiências respiratórias, baixos volumes correntes, com altos fluxos inspiratórios, a fim de ser evitado o auto-PEEP. Como complicações da ventilação mecânica invasiva, temos: pneumonia hospitalar, barotrauma, instabilidade hemodinâmica, autoPEEP, hiperventilação, levando à alcalose respiratória, entre outras.

Medidas adicionais de tratamento, para utilização em pacientes hospitalizados incluem: manutenção do equilíbrio hidroeletrolítico; suporte nutricional adequado, utilizando-se a nutrição suplementar, quando necessária; heparinização profilática em pacientes imobilizados, policitêmicos ou desidratados, com ou sem história de doença tromboembólica; fisioterapia respiratória.

\section{4- Fisioterapia respiratória}

A fisioterapia respiratória tem sido utilizada no tratamento de manutenção e nas exacerbações da DPOC, porém será abordada sua utilização somente nas situações agudas. $\mathrm{O}$ aumento da quantidade de secreção e a disfunção no transporte mucociliar são fatores importantes na fisiopatologia da DPOC, principalmente nos períodos de exacerbação; ambos estão associados com a aceleração do declínio funcional e com o aumento da mortalidade dos pacientes. A retenção de muco é resultado, de um lado, de sua produção excessiva ou da perda de suas propriedades reológicas e, de outro, das dificuldades de sua eliminação, disfunção mucociliar e ineficiência da tosse. De muitas maneiras, intervenções farmacológicas e fisioterápicas podem melhorar o transporte e a eliminação do muco: restabelecendo as propriedades reológicas do muco; estimulando a atividade ciliar; utilizando mecanismos físicos compensatórios, como a gravidade (drenagem postural), interação ar/muco (expiração forçada), vibração, oscilação, percussão e compressão da vias aéreas.

1. Expiração forçada: o princípio terapêutico da manobra baseia-se na premissa de que altos fluxos expiratórios estão associados a aumento do trans- 
porte mucoso. A velocidade do fluxo gasoso faria com que o muco se deslocasse para vias aéreas mais centrais, como resultado da interação e transmissão de energia entre a corrente de ar e as camadas de muco (interação entre fase líquida e gasosa). A efetividade da transmissão de energia depende da velocidade do gás e da espessura da camada de muco, sendo mais eficiente em camadas finas. A compressão das vias aéreas, durante a manobra de expiração forçada, auxilia na eliminação do muco, da mesma forma que a tosse. Entretanto, pacientes podem ter prejuízo na drenagem do muco durante execuções dessas manobras, daí a necessidade de adaptá-las às condições mecânicas do pulmão de pacientes com graves obstruções das vias aéreas.

2. Expansão torácica: hiperinsuflação pulmonar, combinada com compressão externa da caixa torácica são, freqüentemente, utilizadas na prática clínica. Entretanto, existem controvérsias a respeito e de sua segurança e efetividade, particularmente em pacientes cardiopatas, quando os efeitos hemodinâmicos da manobra devem ser considerados.

3. Exercício físico: durante o exercício físico, ocorre aumento da ventilação e liberação de mediadores em vias aéreas, que podem auxiliar no transporte de muco. O exercício associado a outras manobras fisioterápicas aumenta, significativamente, a quantidade de expectoração, mais do que quando utilizado isoladamente.

4. Drenagem postural: durante a drenagem postural, os brônquios maiores são posicionados mais verticalmente, de forma a facilitar a ação das forças gravitacionais sobre o muco, promovendo seu des- locamento para as vias utilizado junto com outros procedimentos fisioterápicos. Considerar que a mudança postural pode afetar a oxigenação, provavelmente, pela interferência na relação ventilação/perfusão pulmonar.

5. Percussão e vibração: a manobra de percussão manual ou mecânica baseia-se na premissa de que as forças vibratórias são transmitidas aos brônquios, facilitando a expectoração. Embora tais oscilações sejam detectadas em brônquios maiores, acreditase que a absorção da força vibratória pela coluna gasosa e pelo parênquima pulmonar atenuem sua atuação em vias aéreas menores. Esta, provavelmente, deve ser a explicação para a ausência de efeitos adicionais de tal manobra sobre o transporte mucoso.

6. Respiração com pressão expiratória positiva: expirar contra uma resistência previne o colapso das vias aéreas e aumenta a ventilação colateral. Sua adição às técnicas de expiração forçada ou drenagem postural aumenta a eliminação de muco. Respiração com lábios semicerrados reproduz, parcialmente, de tal manobra.

Inúmeras outras técnicas podem ser utilizadas em tratamentos mais prolongados e fora da exacerbação, como: exercícios de relaxamento; técnicas de mobilização da caixa torácica; exercício para fortalecimento da musculatura respiratória; treinamento para contrair a musculatura abdominal durante expiração; exercícios respiratórios para otimizar os movimentos toracoabdominais, respiração diafragmática; treinamento/condicionamento da musculatura de membros.

BORGES MC; VIANNA ESO \& TERRA FILHO J. Management in exacerbations of chronic obstructive pulmonary disease (COPD). Medicina, Ribeirão Preto, 36: 241-247, apr./dec. 2003.

ABSTRACT - Exacerbations of chronic obstructive pulmonary disease (COPD) cause increase in morbidity and mortality, hospital admissions, influence health-related quality of life and costs to the patient and health system. COPD patient experience 2 to 3 exacerbations per year, specially during winter, causing a progressive physiologic deterioration and increase in the airway inflammation. Treatment is based on bronchodilators, corticosteroids, oxygen therapy, mechanical ventilation and antibiotics.

UNITERMS - Lung Diseases, Obstructive 


\section{BIBLIOGRAFIA CONSULTADA}

1 - AMERICAN THORACIC SOCIETY. Standards for the Diagnosis and Care of Patients with Chronic Obstructive Pulmonary Disease. Am J Respir Crit Care Med 152: S77-S120, 1995.

2 - ANTHONISEN NR; MANFREDA J \& WARREN CPW. Antibiotic therapy in acute exacerbations of chronic obstructive pulmonary disease. Ann Intern Med 106:196-204, 1987.

3 - BRITISH THORACIC SOCIETY. Guidelines for the management of suspected acute pulmonary embolism. Thorax 58: 470484, 2003.

4 - CLARKE SW; JONES JG \& OLIVER DR. Resistance to twophase gas-liquid flow in airways. J Appl Physiol 29: 464$471,1970$.

5 - COULTAS DB; MAPEL D; GAGNON R\& LYDICK E. The health impact of undiagnosed airflow obstruction in a national sample of United States adults. Am J Respir Crit Care Med 164: 372-377, 2001.

6 - GOSSELINK R. Principles of physiotherapy. In GIBSON GJ, ed. Respiratory medicine, $3^{\mathrm{a}}$ ed. Saunders, Philadelphia. p. 554-562, 2003.

7 - HASAM A; PAVIC D; AGREW JE \& CLARKE SW. Regional lung clearance during cough and forced expiration technique(FET): effects of flow and viscoelasticity. Thorax 49: 557-561, 1994.

8 - MURRAY CJ \& LOPEZ AD. Alternative projections of mortality an disability by cause 1990-2020: Global Burden of Disease Study. Lancet 349: 1498-1504, 1997.

9 - NIEWOEHNER DE. The role of systemic corticosteroids in acute exacerbation of chronic obstructive pulmonary disease. Am J Respir Med 1: 243-248, 2002.

10 - PAUWELS RA; BUIST AS; CALVERLEY PM; JEWKINS CR; HURD SS \& GOLD SCIENTIFIC COMMITTEE. Global strategy for the diagnosis, management, and prevention of chronic obstructive pulmonary disease: NHLBI/WHO Global Initiative for Chronic Obstructive Lung Disease (GOLD) workshop summary. Am J Resp Crit Care Med 163:1256-1276, 2001.
11 - PLANT PK\& ELLIOTT MW. Chronic obstructive pulmonary disease: Management of ventilatory failure in COPD. Thorax 58: 537-542, 2003.

12 -PLANT PK; DWEN JL\& ELLIOTT MW. Early use of non-invasive for acute exacerbations of chronic obstructive pulmonary disease on general respiratory wards: a multicentre randomized controlled trial. Lancet 335:1931-1935, 2000.

13 - I CONSENSO BRASILEIRO DE DOENÇA PULMONAR OBSTRUTIVA CRÔNICA (DPOC). J Pneumol 26: S1-S52, 2000.

14 - RAM FS; PICOT J; LIGHTOWOLER J\& WEDZICHA JA. Noninvasive positive pressure ventilation for treatment of respiratory failure due to exacerbations of chronic obstructive pulmonary disease. Cochrane Database Syst Rev (1):CD004104, 2004.

15 -SIN DD; MCALISTER FA; MAN SFP\& ANTHONISEN NR. Contemporary Management of Chronic Obstructive Pulmonary Disease. JAMA 290: 2301-2312, 2003.

16 - THE PIOPED INVESTIGATORS. Value of the ventilation/perfusion scan in acute pulmonary embolism: results of the prospective investigation of pulmonary embolism diagnosis. JAMA 263:2753-2759, 1990.

17 - VESTBO G; PRESCOTT E; LANGE PAND THE COPENHAGEN CITY HEART STUDY GROUP. Association of chronic mucus hypersecretion with FEV1 decline and chronic obstructive pulmonary disease morbidity. Am J Respir Crit Care Med 153:1530-1535, 1996.

18 - WEDZICHA JÁ \& DONALDSON GC. Exacerbations of chronic obstructive pulmonary disease. Respir Care 48: 120412013, 2003.

19 - WOOD KA; LEWIS L; VON HONG B \& KOLLEF MH. The use of non-invasive positive pressure ventilation in the Emergency Department. Chest 113:1339-1346, 1998. 